\title{
No effectiveness of synthetic anti-inflammatory tetrapeptides in a mouse model of ischemic stroke
}

\author{
Heleen M den Hertog ${ }^{1}$, Karen Gertz ${ }^{2,3}$, Wim A Dik ${ }^{4}$, Dirk J Duncker ${ }^{5}$, Nisar A Khan ${ }^{4}$, Matthias Endres ${ }^{2,3}$, \\ Robbert Benner ${ }^{4}$ and Peter J Koudstaal ${ }^{1}$ \\ Departments of ${ }^{1}$ Neurology, ${ }^{4}$ Immunology, and ${ }^{5}$ Cardiology, Erasmus University Medical Center, Rotterdam, The \\ Netherlands \\ ${ }^{2}$ Department of Neurology, ${ }^{3}$ Center for Stroke Research Berlin, Charité Universitätsmedizin Berlin, Germany
}

\begin{abstract}
Background. Inflammation plays an important role in the pathophysiology of ischemic stroke and may contribute to secondary damage following ischemic stroke. Recently, it has been shown that synthetic oligopeptides related to human chorionic gonadotropin (hCG) and various other synthetic oligopeptides may have immunomodulatory effects.
\end{abstract}

We aimed to investigate the effects of two promising synthetic anti-inflammatory tetrapeptides, namely the hCGrelated peptide AQGV and the p53-related peptide EPPE on infarct volume and on inflammatory gene expression in a mouse model of focal cerebral ischemia.

Methods. In a randomized single-blinded fashion, mice received two intravenous injections of either AQGV (30 $\mathrm{mg} / \mathrm{kg}$ bodyweight) or EPPE (30mg/kg bodyweight) or phosphate buffered saline. The first dose was administered directly before 90-minutes middle cerebral artery occlusion, and the second dose was injected directly after reperfusion. Infarct volume and gene expression levels of tumor necrosis factor- $\alpha$ (TNF- $\alpha$ ), interleukin-6 (IL6), E-selectin and intercellular adhesion molecule-I (ICAM-1) were measured 24 hours after reperfusion.

Results. We found no significant effect of either AQGV $\left(108.7+/-12.1 \mathrm{~mm}^{3}\right.$ versus PBS $114.9+/-23.9 \mathrm{~mm}^{3}$; $\mathrm{P}=0.7)$ or EPPE $\left(131.8+/-12.8 \mathrm{~mm}^{3}\right.$ versus PBS $\left.114.9+/-23.9 \mathrm{~mm}^{3} ; \mathrm{P}=0.1\right)$ on direct lesion volume. All three experimental groups displayed similar mRNA expression levels of TNF- $\alpha$, IL-6, E-selectin and ICAM-1 at 24 hours after the ischemic insult.

Conclusion. AQGV and EPPE did not show any beneficial effects in this mouse model of acute ischemic stroke. Further studies are needed to investigate the nature and dynamics of the immunomodulatory effects of synthetic oligopeptides in acute ischemic stroke.

Keywords: Ischemic stroke, inflammation, immunomodulation

\section{Introduction}

Inflammation plays a central role in the pathophysiology of ischemic stroke. In the early phase of ischemia and reperfusion, an inflammatory cascade is set off in the ischemic core and in the penumbra, where perfusion is compromised but tissue is still viable (Del Zoppo et al, 2001). Numerous pro-inflammatory genes are upregulated, including those for transcription factors, heat shock proteins, cytokines, chemokines and adhesion molecules. The classical proinflammatory cytokines interleukin-6 (IL-6), and tumor necrosis factor- $\alpha$ (TNF- $\alpha$ ), produced by microglia, astrocytes, endothelial cells, and neurons, mediate the inflammatory processes (Wang et al, 2007). They activate leucocytes, stimulate the release of chemokines, and increase adhesion molecule expression on cerebral microvessels and circulating leukocytes, including intercellular adhesion molecule (ICAM-1) and E-selectin (Wang et al, 2007). The early inflammatory phase is followed by a tissue-remodelling phase during which tissue-remodelling factors like MMP9, MMP2 and TGF- $\beta$ are expressed and is associated with healing of the ischemic brain tissue. The inflam-

* Correspondence should be sent to:

Heleen M den Hertog, M.D., Ph.D.; Department of Neurology, Erasmus University Medical Center, PO Box 2040, 3000 CA Rotterdam,

The Netherlands; Email address: m.denhertog@erasmusmc.nl ; Telephone: +31107040704; Fax: +31107044721. 
matory processes start within 2 hours after stroke onset and sustain for several days. Besides its beneficial effect, inflammation may contribute to the secondary progression of ischemic brain injury. The acute inflammatory phase is believed to be detrimental, while the chronic phase inflammation may be essential for repair and regeneration (Del Zoppo et al, 2001; Barone et al, 1999; Bowen et al, 2006). This is a concept that holds promise for new therapeutic interventions.

The adaptations of the immune system during pregnancy are striking. The maternal immune system is under tight control to prevent rejection of the foetal allograft. This suggests that a specific hormonal environment is responsible for modulating the immune system during pregnancy. Human chorionic gonadotropin (hCG) is secreted by placental syncytiotrophoblasts, but is also produced by the pituitary gland and leucocytes in non-pregnant females and males (Benner et al, 2005; Birken et al, 1996; Yoshimoto et al, 1979). HCG consists of an $\alpha$ - and a $\beta$-chain. In human pregnancy urine, hCG occurs in a variety of forms, including oligopeptidic breakdown products of the $\alpha \beta$-chain (Benner et al, 2005). HCG preparations have been recognized to exert immunomodulatory activities that are not due to the native molecule nor to it's $\alpha$ - and $\beta$-chain, but resided in specific peptide fractions (Benner et al, 2005). Based on known preferential cleavage sites, several oligopeptides from $\beta$ hCG residues have been synthesized. These oligopeptides including AQGV have shown promise in the treatment of ischemia-reperfusion injury such as septic shock (Khan et al, 2002), hemorrhagic shock (van den Berg et al, 2009) and renal ischemia-reperfusion injury (Khan et al, 2009). Several regulatory oligopeptides based on the primary sequence of other proteins have also been generated and proven biologically active in different models tested. One of these regulatory oligopeptides is the p53-related tetrapeptide EPPE.

In this study, as a first step in assessing potential beneficial effects of synthetic anti-inflammatory tetrapeptides in acute ischemic stroke, we investigated the effects of the $\beta$-hCG related synthetic tetrapeptide AQGV and the p53-related synthetic tetrapeptide EPPE on infarct volume and on inflammatory gene expression in an animal model of focal cerebral ischemia.

\section{Methods}

\subsection{Animals}

Experiments were performed in male 129S6/SvEv mice, weighing $20-30 \mathrm{~g}$, in accordance with the $\mathrm{NIH}$ Guidelines for the Care and Use of Laboratory Animals and with approval of the local Animal Care Committee.

\subsection{Synthetic anti-inflammatory tetrapeptides}

$\beta$-hCG-related AQGV showed the most powerful protection in a model of renal ischemia-reperfusion injury (Khan et al, 2009). Since there are many similarities in the pathophysiology of acute cerebral ischemiareperfusion injury and acute renal ischemiareperfusion injury, we decided to study the effects of AQGV, and another promising p53-related tetrapeptide EPPE in our model of focal cerebral ischemia.

AQGV and EPPE were synthesized (Ansynth Service BV Roosendaal, the Netherlands) using the fluorenylmethoxycarbonyl (Fmoc)/tert-butyl-based methodology with a 2-chlorotritylchloride resin as the solid support and dissolved in $0.9 \%$ sodium chloride at a concentration of $30 \mathrm{mg} / \mathrm{ml}$.

\subsection{Model of focal cerebral ischemia}

Anesthesia was induced by $1.5 \%$ halothane and maintained with 1\% halothane in $70 \% \mathrm{~N}_{2} \mathrm{O}$ and $30 \%$ $\mathrm{O}_{2}$. Ischemia was induced by filamentous occlusion of the left middle cerebral artery for 90 minutes as described previously (Gertz et al, 2003). In brief, brain ischemia was induced with an 8.0 nylon monofilament coated with a silicone resin/hardener mixture (Xantopren M Mucosa and Activator NF Optosil Xantopren, Haereus Kulzer, Germany). The filament was introduced into the left common carotid artery up to the anterior cerebral artery. Thereby the middle cerebral artery and anterior choroidal arteries were occluded. Filaments were withdrawn after 90 minutes to allow reperfusion. The core temperature of the mice during the procedure was maintained at $36.5^{\circ} \mathrm{C}$ using a feedback temperature control unit.

\subsection{Administration of synthetic anti-inflammatory te- trapeptides}

A previous dose-escalating study of AQGV in a model of renal ischemia-reperfusion injury showed that AQGV was effective in doses above $0.3 \mathrm{mg} / \mathrm{kg}$ bodyweight (Khan et al, 2009). For this study, we determined the effect of the highest tested dose in the dose escalating study, namely $30 \mathrm{mg} / \mathrm{kg}$ bodyweight.

Three groups of mice (10 mice per group) received, in a randomized single- blinded fashion, either AQGV (30 $\mathrm{mg} / \mathrm{kg}$ bodyweight) or EPPE (30 $\mathrm{mg} / \mathrm{kg}$ bodyweight) or $1 \mathrm{ml}$ phosphate buffered saline (PBS), which was administered intravenously in two slow injections ( $0.1 \mathrm{ml}$ over 5 minutes). Administration of the first dose was started directly before middle cerebral artery occlusion (MCAO). The second dose was administered directly after reperfusion.

\subsection{Measurement of ischemic lesion size}

The animals were sacrificed 24 hours after reperfusion. The brains were snap-frozen in isopentane for cryostat sectioning. Infarct areas were quantitated by -34 -

J Exp Stroke Transl Med (2010) 3(1): 33-38

Society for Experimental Stroke (www.s4es.org) 
use of sigma 4.0 software (sigmaScan Pro 4.0, Jandel scientific) on 20- $\mu \mathrm{m}$ hematoxylin-and eosinstained cryostat sections without knowledge of treatment. Direct infarct volumes were calculated by summing the lesion volumes of each section (we used direct lesion size) $\left(\mathrm{mm}^{3}\right)$.

2.6 Evaluation of mRNA levels by real-time quantitative polymerase chain reaction

RNA was isolated from $20-\mu \mathrm{m}$ cryostat sections with a Qiagen RNeasy kit (QIAGEN, Hilden, Germany) according to the manufacturer's instructions, and reverse transcribed into cDNA. We determined gene expression levels of TNF- $\alpha$, IL-6, E-selectin and ICAM-I with Applied Biosystems 7700 PCR machine (Foster City, California), as described previously. The expression levels of these genes were quantified by normalization against the mRNA levels of the household gene ABL.

\subsection{Data analysis and presentation}

Statistical analyses were performed using SPSS version 11 software (SPSS, inc., Chicago III). Data are presented as mean values +/- SD of the ten mice per group. Comparisons were made by one-way of variance (ANOVA), followed by Duncan's multiple range test.

\section{Results}

Of the total 30 mice entered in the study, two mice in the EPPE group and one mouse in the AQGV group died, because of technical failure during surgery.

\subsection{AQGV and EPPE do not alter infarct volume}

The direct infarct volume was not significantly different between AQGV-treated mice and mice treated with PBS $\left(108.7+/-12.1 \mathrm{~mm}^{3}\right.$ versus $114.9+/-23.9$ $\left.\mathrm{mm}^{3} ; \mathrm{P}=0.7\right)$ and between EPPE-treated and mice treated with PBS $\left(131.8+/-12.8 \mathrm{~mm}^{3}\right.$ versus 114.9 +/- $23.9 \mathrm{~mm}^{3} ; \mathrm{P}=0.1$ ) (Fig.1).

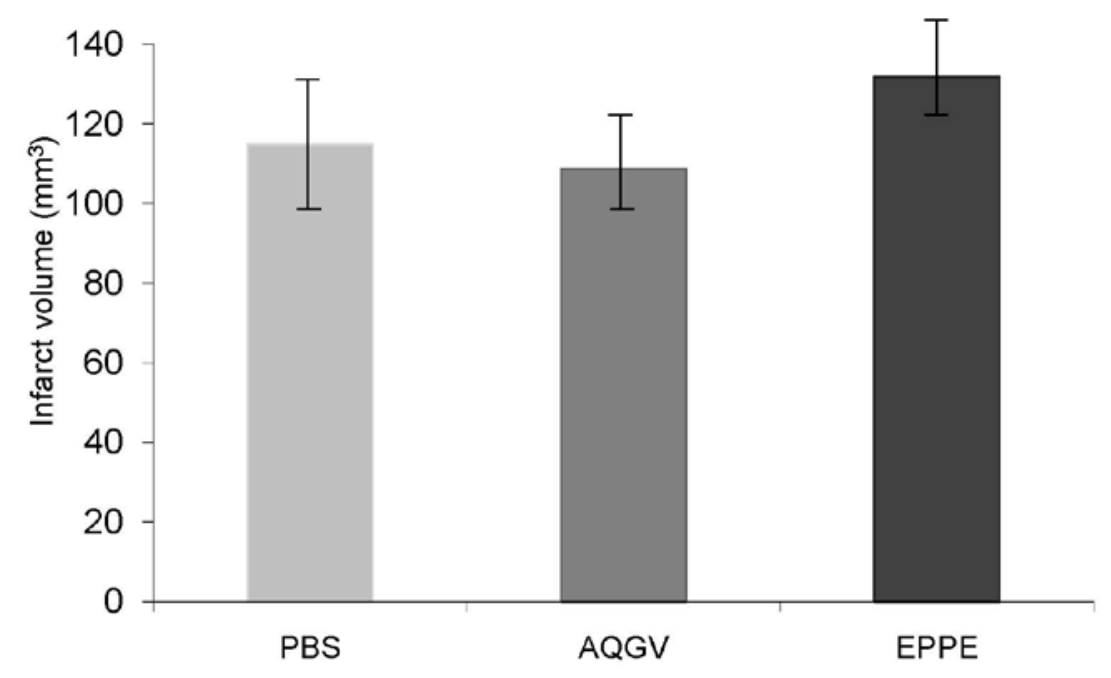

Fig. 1. Effects of treatment with AQGV and EPPE versus PBS on mean direct ischemic lesion volume (mm3) +/- SD.

3.2 AQGV and EPPE do not influence mRNA transcription levels of TNF- $\alpha$, IL-6, ICAM-1 and E-selectin

All three experimental groups displayed comparable mRNA expression levels of TNF- $\alpha$, IL-6, ICAM-1 and
E-selectin at 24 hours after the ischemic insult (Fig. $2 \mathrm{~A}$ and $2 \mathrm{~B})$. 

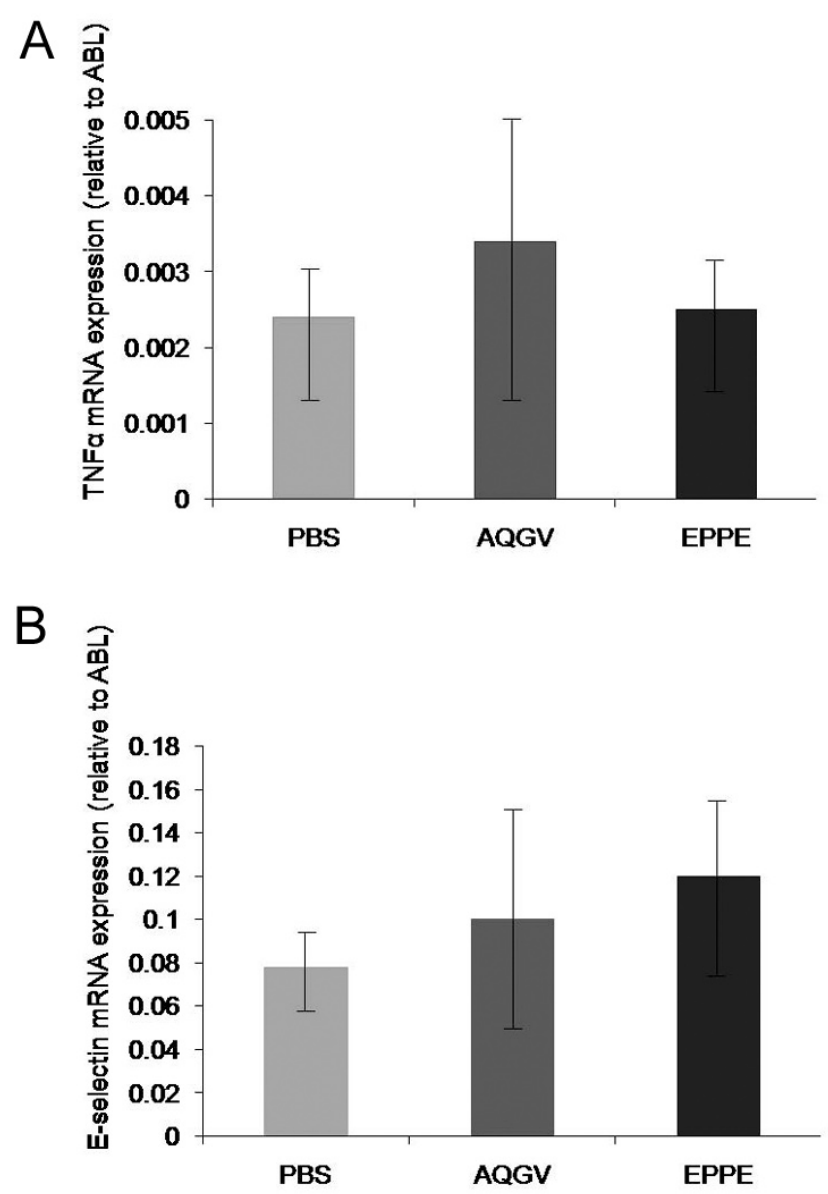
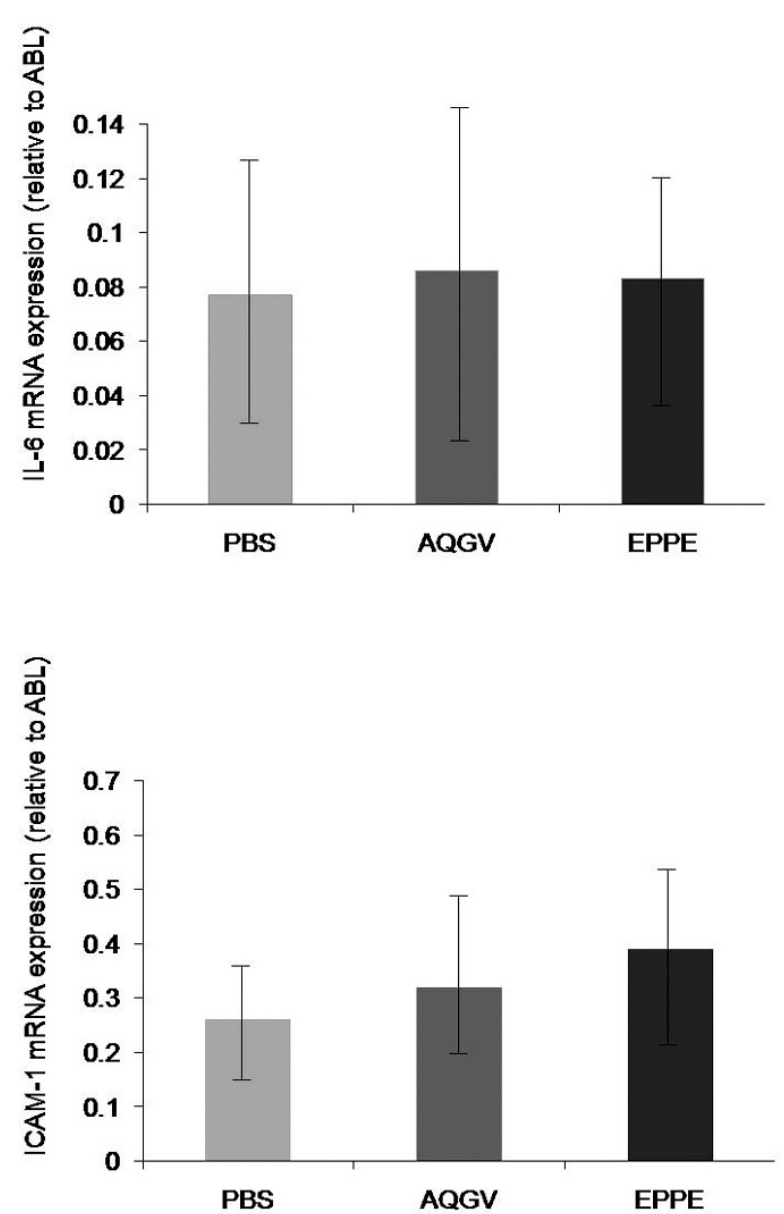

Fig 2. Relative expression of mRNA transcription levels of pro-inflammatory cytokines TNF- $\alpha$ and IL-6 (A) and adhesion molecules E-selectin and ICAM-1 (B) in the brain 24 hours after reperfusion. Transcription levels are normalized to the expression level of the household gene ABL. Data are presented as the mean of per group $+/$ SD.

*Neither AQGV nor EPPE caused significant differences in the transcription levels of TNF- $\alpha$, IL-6, E-selectin and ICAM-1 as compared with PBS.

\section{Discussion}

In this study, we found that in an established mouse model of ischemic stroke, treatment with two synthetic anti-inflammatory tetrapeptides had no effect on infarct volume or on mRNA expression levels of adhesion molecules and pro-inflammatory cytokines.

Our results deviate from the beneficial effects of treatment with synthetic anti-inflammatory oligopeptides in various other in vivo models of ischemiareperfusion injury (Khan et al, 2002; van den Berg et al, 2009; Khan et al, 2009). Treatment with such synthetic anti-inflammatory oligopeptides has been shown to inhibit renal ischemia-reperfusion injury and to increase survival rates by reducing systemic inflammation and adhesion molecule expression in the kidney (Khan et al, 2009). These peptides also atte- nuated inflammation and liver damage after hemorrhagic shock (van den Berg et al, 2009) and decreased morbidity and mortality associated with lipopolysaccharide injection (Khan et al, 2002).

There are several explanations why no such beneficial effects of treatment with synthetic antiinflammatory oligopeptides were found in our model of acute ischemic stroke. First, the role of inflammation in the acute phase of stroke is ambivalent. The inflammatory reaction following ischemic stroke triggers the removal of noxious agents and supports tissue cleaning and repair. Contrary to these beneficial effects, it may augment secondary damage by disruption of the blood-brain barrier, release of cytotoxic agents, edema resulting from endothelial cell injury and leucocyte-mediated injury, increased body temperature and microvascular thrombosis. Since the underlying mechanisms by which the synthetic anti- 
inflammatory oligopeptides exert their effects are unclear, it cannot be excluded that they might also negate the positive effects of the inflammatory reaction. On the other hand, synthetic anti-inflammatory oligopeptides did show beneficial effects in renal ischemia-reperfusion injury and there are many similarities in the pathophysiology of acute cerebral ischemiareperfusion injury and acute renal ischemiareperfusion injury (Khan et al, 2009).

Furthermore, we did not compare the mRNA expression levels of adhesion molecules and proinflammatory cytokines with those in brains of shamoperated animals. Therefore, we do not know whether these pro-inflammatory genes were upregulated in our model of ischemic stroke. However, there is substantial evidence from previous studies that these genes are upregulated witin the first hours of stroke onset (Del Zoppo et al, 2001; Wang et al, 2007; Barone et al, 1999).

It should also be noted that different synthetic antiinflammatory oligopeptides might have different modes of action. This is supported by previous studies that have identified different synthetic anti-inflammatory oligopeptides as the most effective mediator (Khan et al, 2002; van den Berg et al, 2009; Khan et al, 2009).

Another explanation of the negative results of our study is that it was not designed to investigate different doses of AQGV or EPPE. Therefore, we cannot rule out that there is a dose-dependent effect, as was observed in previous studies (Khan et al, 2002; van den Berg et al, 2009; Khan et al 2009). Also, the two time-points of peptide administration chosen for the current study may not have been optimal. Neither can we exclude that repeated peptide administrations during the study period could have been effective.

We have studied only the effects of AQGV and EPPE on infarct volume and mRNA transcription levels at 24 hours after reperfusion. As the inflammatory reaction is initiated within a few hours after stroke onset and lasts for several days, it may be worthwhile to monitor the early evolution of brain injury at different points in time with MRI scanning (Hoehn et al, 2001). The major advantage of MRI over histologic examinations is its potential to non-invasively assess changes in the brain in vivo. Finally, since no measurements of brain concentrations of the administered tetrapeptides were performed we do not know whether the oligopeptides underwent presystemic transformation and actually crossed the blood-brain barrier. However, a previous study showed that hCG crossed the blood-brain barrier in an intact form in rats (Lukacs et al, 1995). Furthermore, their small size as well as the disruption of the blood-brain barrier following ischemic stroke makes it likely that tetrapeptides did cross the blood-brain barrier.
HCG is not the only protein source for regulatory oligopeptides. Sequences of other proteins such as Creactive protein Ig heaving chain, phospholipase $A 2$, hemoglobin, b-defensins, lactoferrin and granulysin possess anti-inflammatory activities with the function being expressed upon the degradation of the parent protein (King et al, 2002; King et al, 2003; King et al 2007; Miele et al 1988; Parish et al, 2001; Rawlings et al, 2008; Robey et al, 1987). So far about 500 human genes encoding proteases/peptidases have been identified. This might be related to regulation of biological processes by peptidases and might imply that virtually every protein within the body can serve as a source of regulatory oligopeptides.

Our results do not negate the possibility that antiinflammatory oligopeptides might be useful for acute stroke treatment. Further studies are needed to investigate the nature and dynamics of the immunomodulatory effects of synthetic anti-inflammatory oligopeptides in acute ischemic stroke.

\section{Conclusion}

AQGV and EPPE did not show any beneficial effects in this mouse model of acute ischemic stroke. Further studies are needed to investigate the nature and dynamics of the immunomodulatory effects of synthetic oligopeptides in acute ischemic stroke.

\section{Conflict of Interest}

None

\section{References}

Barone FC, Feuerstein GZ. Inflammatory mediators and stroke: new opportunities for novel therapeutics (1999). J Cereb Blood Flow Metab 19:819-834.

Benner R, Khan NA. Dissection of systems, cell populations and molecules (2005). Scand J Immunol 62 Suppl 1:62-66.

Birken S, Maydelman Y, Gawinowicz MA, Pound A, Liu Y, Hartree AS. Isolation and characterization of human pituitary chorionic gonadotropin (1996). Endocrinology 137:1402-1411.

Bowen KK, Naylor M, Vemuganti R. Prevention of inflammation is a mechanism of preconditioning-induced neuroprotection against focal cerebral ischemia (2006). Neurochem Int 49:127-135.

Del Zoppo GJ, Becker KJ, Hallenbeck JM. Inflammation after stroke: is it harmful? (2001) Arch Neuro 58:669672 .

Dik WA, Nadel B, Przybylski GK, Asnafi V, Grabarczyk P, Navarro JM, Verhaaf B, Schmidt CA, Macintyre EA, van Dongen JJ, Langerak AW. Different chromosomal breakpoints impact the level of LMO2 expression in TALL (2007). Blood 110:388-392. 
Gertz K, Laufs U, Lindauer U, Nickenig G, Bohm M, Dirnagl $\mathrm{U}$, Endres M. Withdrawal of statin treatment abrogates stroke protection in mice (2003). Stroke 34:551-557.

Hoehn M, Nicolay K, Franke C, van der Sanden B. Application of magnetic resonance to animal models of cerebral ischemia (2001). J Magn Reson Imaging 14:491509.

Khan NA, Khan A, Savelkoul HF, Benner R. Inhibition of septic shock in mice by an oligopeptide from the betachain of human chorionic gonado- trophin hormone (2002). Hum Immunol 63:1-7.

Khan NA, Susa D, van der Berg JWI. Amelioration of renal ischemia reperfusion injury by synthetic oligopeptides related to human chorionic gonadotropin (2009). Nephrology Dialysis Transplantation 24:2701-2708.

King AE, Fleming DC, Critchley HO, Kelly RW. Regulation of natural antibiotic expression by inflammatory mediators and mimics of infection in human endometrial epithelial cells (2002). Mol Hum Reprod 8:341-349.

King AE, Critchley HO, Sallenave JM, Kelly RW. Elafin in human endometrium: an antiprotease and antimicrobial molecule expressed during menstruation (2003). J Clin Endocrinol Metab 88:4426-4431.

King AE, Kelly RW, Sallenave JM, Bocking AD, Challis JR. Innate immune defences in the human uterus during pregnancy (2007). Placenta 28:1099-1106.

Lukacs H, Hiatt ES, Lei ZM, Rao CV. Peripheral and intracerebroventricular administration of human chorionic gonadotropin alters several hippocampus-associated behaviors in cycling female rats (1995). Horm Behav 29:42-58
Miele L, Cordella-Miele E, Facchiano A, Mukherjee AB. Novel anti-inflammatory peptides from the region of highest similarity between uteroglobin and lipocortin I (1998). Nature 335:726-730.

Parish CA, Jiang $\mathrm{H}$, Tokiwa $\mathrm{Y}$, Berova $\mathrm{N}$, Nakanishi $\mathrm{K}$, McCabe D, Zuckerman W, Xia MM, Gabay JE (2001). Broad-spectrum antimicrobial activity of hemoglobin. Bioorg Med Chem 9:377-382.

Rawlings ND, Morton FR, Kok CY, Kong J, Barrett AJ. MEROPS: the peptidase database (2008). Nucleic Acids Res 36:D320-D325.

Robey FA, Ohura K, Futaki S, Fujii N, Yajima H, Goldman $\mathrm{N}$, Jones KD, Wahl S. Proteolysis of human C-reactive protein produces peptides with potent immunomodulating activity (1987). J Biol Chem 262:7053-7057.

van den Berg HR, Khan NA, van der ZM, Bonthuis F, IJzermans JN, Dik WA, de Bruin RW, Benner R. Synthetic oligopeptides related to the [beta]-subunit of human chorionic gonadotropin attenuate inflammation and liver damage after (trauma) hemorrhagic shock and resuscitation (2009). Shock 31:285-291.

Wang Q, Tang XN, Yenari MA. The inflammatory response in stroke (2007). J Neuroimmunol 184:53-68.

Yoshimoto $Y$, Wolfsen AR, Hirose F, Odell WD. Human chorionic gonadotropin--like material: presence in normal human tissues (1979). Am J Obstet Gynecol 134:729-33. 